\title{
Coupled clumped isotope analysis on the Nu Perspective IS
}

\author{
Q. LI $^{1}$, P. FREEDMAN ${ }^{1}$
}

${ }^{1} \mathrm{Nu}$ Instruments Ltd, Unit 74, Clywedog Road South, Wrexham, LL13 9XS UK

The combined $\Delta_{47}$ and $\Delta_{48}$ thermometry provides an important analytical approach for reliable formation temperature estimates of carbonate systems. Here we present both $\Delta_{47}$ and $\Delta_{48}$ measurements on a $\mathrm{Nu}$ Perspective $I S$ stable isotope ratio mass spectrometer using the method of Fiebig et al. (2019). We compare the precisions of the clumped dataset of this study and that of Fiebig et al. (2019); and discuss the use of $10^{13} \Omega$ on clumped isotope analysis.

The Perspective $I S$ is coupled to an automated Nucarb carbonate prep unit. Each analysis uses only $4-5 \mathrm{mg}$ of pure carbonate standard which was dessolved in $1.94 \mathrm{~g} / \mathrm{ml}$ phosphoric acid at $70{ }^{\circ} \mathrm{C}$ to evolve $\mathrm{CO}_{2}$ gas. The gas was then separated cryogenically and purified through a Porapak trap before being measured for masses 44-49. Data acquisition consists of 100 cycles, each of 20 s integration time; the mass 44 signal is $16 \mathrm{~V}$ with a $2 \times 10^{8} \Omega$ resistor. Clumped isotope collectors (masses 47-49) were fitted with $10^{12} \Omega$ resistors and mini-ESAs to remove background noise.

A total number of 140 measurements of 5 carbonate standards (NCM, ETH-1 to 4) were conducted over JanuaryFebruary 2020. The raw $\Delta_{47}$ and $\Delta_{48}$ and the number of analyses are listed in the table below. The external precision (1SD) is typically $0.01 \%$ on $\Delta_{47}$ and between $0.03-0.04 \%$ on $\Delta_{48}$; this is very similar to that in Fiebig et al. (2019), whose system employs a customer-built prep line that takes $10 \mathrm{mg}$ of sample each run and uses $10^{13} \Omega$ resistors for the clumped isotopes and half mass 47.5 collector for baseline correction. In contrast, the Nucarb only uses half of the sample size per analysis with $10^{12} \Omega$ resistors.

\begin{tabular}{|c|c|c|c|c|c|}
\hline This Study & $\Delta_{47}$ raw & $1 s d$ & $\Delta_{48}$ raw & $1 s d$ & $N$ \\
\hline NCM & -0.591 & 0.009 & -0.323 & 0.040 & 58 \\
\hline ETH-1 & -0.682 & 0.014 & -0.328 & 0.032 & 20 \\
\hline ETH-2 & -0.680 & 0.009 & -0.148 & 0.037 & 18 \\
\hline ETH-3 & -0.300 & 0.013 & -0.201 & 0.042 & 22 \\
\hline ЕTH-4 & -0.456 & 0.015 & -0.079 & 0.037 & 22 \\
\hline
\end{tabular}

Our results show that with such large sample size, $10^{13} \Omega$ resistors are not expected to improve the precision. This is because the uncertainties on masses 47 and $48(\sim 4 \mathrm{~V}$ and $0.4 \mathrm{~V}$ on $10^{12} \Omega$ resistors) are dominated by shot noise (associated with counting statistics), but not Johnson noise (caused by resistors). Further tests will be performed on Perspective $I S$ with $10^{13} \Omega$ resistors and smaller samples.

[1] Fiebig et al. (2019) Chem. Geol. 522, 186-191. 